\title{
Effect of the Representative Source Area for Eddy Covariance Measuraments on Energy Balance Closure for Maize Fields in the Po Valley, Italy
}

\author{
Daniele Masseroni", Chiara Corbari, Marco Mancini \\ Dipartimento di Ingegneria Idraulica, Ambientale, Infrastrutture Viarie e Rilevamento, Politecnico di Milano, P.zza Leonardo da Vinci 32, \\ 20133 Milano, Italy
}

\begin{abstract}
This paper has as main objective to show the effect of the representative source area for eddy covariance measurements (called footprint) on energy balance closure. Energy balance closure was evaluated by a statistical regression of turbulent energy fluxes (sensible and latent heat) against available energy (net radiation and soil ground heat flux). The footprint was calculated using an approximate analytical model based on a combination of Lagrangian stochastic dispersion model and dimensional analysis. The data were measured by two eddy covariance towers located on maize fields in Landriano (PV) and Livraga (LO) at the Po Valley, Italy. The main results obtained using only the flux data which have a source area included into the cultivated field shows that there is a slight improvement on the energy balance closure. The stability conditions of the atmosphere plays a fundamental role on the slope of the linear regression and on footprint size, in particular way, it is shown when the energy balance closure is analysed for different sectors of the field in function of the wind directions.
\end{abstract}

Keywords Footprint, Energy Balance Closure, Eddy Covariance Method

\section{Introduction}

The eddy covariance technique is generally used to estimate turbulence fluxes of mass and energy to and from surfaces. A difficult but important issue remains to quantify estimates of the uncertainty of the reported flux values. These fluxes are in fact complex processes, and the estimates result from various measurements and calculations as well as numerous explicit and implicit assumptions[4,23]. Hence, documenting the absolute accuracy of these values is somewhat problematic. However, one simple measure of internal consistency is to check for conservation of energy. So the sum of the turbulence fluxes of sensible and latent heat should balance the available energy[9]. The surface energy balance may be written as in (1).

$$
\text { closure }=\frac{L E+H}{R_{n}-G}
$$

Where $R_{n}$ is net radiation, $G$ is soil heat flux, $H$ is the sensible heat flux and $L E$ is latent heat flux. In a perfect world, measurements would be perfect and the closure value would be close to 1 .

The source area of a turbulent flux defines the spatial

* Corresponding author:

daniele.masseroni@mail.polimi.it (Daniele Masseroni)

Published online at http://journal.sapub.org/ijaf

Copyright (C) 2011 Scientific \& Academic Publishing. All Rights Reserved context of the measurement. It is something akin to the "field of view" of the measurement of surface atmosphere exchange. When turbulent flux sensors are deployed, the objective is usually to measure signals that reflect the influence of the underlying surface on the turbulent exchange. The measured signal depends on which part of the surface has the strongest influence on the sensor, and thus on the location and size of its footprint[17]. The footprint size can be considered like a representative area of the sensor detector. Generally the footprint can be represented like a distance from the tower (where sensors detector are located) upwind the preferential direction of the wind velocity. Many models are available to calculate the footprint dimension; for example the Lagrangian, Eulerian and LES (Large Eddy Simulation) models are very complicated but they try to represent the conditions of the atmospheric turbulence in a realistic way[5,11,12,14,15,21]. One of the problems of these models is the computational cost required to solve equations. A simple model based on the combination of Lagrangian stochastic model and dimensional analysis is represented by the Hsieh's hybrid model[10]. The advantage of this model is that it can be applied in neutral, stable and unstable conditions and compared with Gash's Eulerian model[8] and Thomson's Lagrangian model[20] results, produces a correct assess of the footprint size.

There are several reasons because the balance closure value is not perfectly $1[7]$, but two principal problems are: 
the dimension of source area for eddy fluxes measurements and stability conditions of the atmosphere. The objective of this work is to show how the energy balance closure changes using only flux measurements with a source area equal to the field size. Moreover, it is shown how the slope values of the linear regression for energy balance closure and the footprint dimension changes according to the different stability conditions of the atmosphere (unstable, neutral e stable conditions).

\section{Theoretical Background}

\subsection{Eddy Covariance Technique}

The turbulence of the air flow can be described, using the Reynold's hypothesis, subdividing wind speed and the scalar quantities in a mean component and in a fluctuant component (2).

$$
\begin{aligned}
& U(x, y, z, t)=\bar{U}(x, y, z, t)+u^{\prime}(x, y, z, t) \\
& V(x, y, z, t)=\bar{V}(x, y, z, t)+v^{\prime}(x, y, z, t) \\
& W(x, y, z, t)=\bar{W}(x, y, z, t)+w^{\prime}(x, y, z, t) \\
& T(x, y, z, t)=\bar{T}(x, y, z, t)+T^{\prime}(x, y, z, t) \\
& q(x, y, z, t)=\bar{q}(x, y, z, t)+q^{\prime}(x, y, z, t)
\end{aligned}
$$

The $U, V, W$ are the instantaneous three-dimensional components of the wind speed, $T$ is the scalar temperature and $q$ is the scalar concentration of vapour in air. $\bar{U}, \bar{V}, \bar{W}, \bar{T}$ and $\bar{q}$ represents the mean components and the $u^{\prime}, v^{\prime}, w^{\prime}, T^{\prime}$ and $q$ ' represents the fluctuant components. It is important to remark that these components are a function of the spatial position $(x, y, z)$ and time $(t)$.

The eddy covariance method determines the surface fluxes as the sum of turbulent eddy-fluxes, measured above the surface, and the flux divergence between the surface and the eddy covariance measurement level[2]. The basic equations to estimate latent and sensible heat fluxes, (3) and (4), are comparatively simple.

$$
\begin{aligned}
& L E=\lambda \rho \overline{w^{\prime} q^{\prime}} \\
& H=\rho C_{p} \overline{w^{\prime} T^{\prime}}
\end{aligned}
$$

Where $\lambda$ is the vaporization latent heat, $\rho$ the air density and $\overline{w^{\prime} q^{\prime}}$ the covariance between vertical wind velocity component and scalar concentration of vapor in the air. $C_{p}$ is the specific heat at constant pressure and $\overline{w^{\prime} T^{\prime}}$ is the covariance between vertical wind velocity component and scalar temperature.

The covariance of the vertical wind velocity and scalar quantities can be determined by (5):

$$
\overline{w^{\prime} \varepsilon^{\prime}}=\frac{1}{N-1} \sum_{k=1}^{N-1}\left[\left(w_{k}-\bar{w}_{k}\right)\left(\varepsilon_{k}-\bar{\varepsilon}_{k}\right)\right]
$$

Where $\varepsilon$ represents a generic scalar quantity and $N$ the total number of data.

The energy balance closure can be written as (6):

$$
L E+H=R_{n}-G
$$

$R_{n}$ and $G$ can be calculated using respectively equation (7) and (8).

$$
\begin{gathered}
R_{n}=R_{l_{-} i}+R_{s_{-} i}-R_{l_{-} o}-R_{s_{-} o} \\
G=a_{G} \frac{\Delta T_{s}}{\Delta z}
\end{gathered}
$$

The net radiation in the atmosphere is divided into shortwave radiation and long wave radiations. The net radiation is the sum of the shortwave down welling radiation mainly from the sun $\left(R_{S_{-}}\right)$, the long wave dowelling infrared radiation emitted by clods, aerosol and gases $\left(R_{l i}\right)$, the shortwave up-welling reflected radiation $\left(R_{s_{o}}\right)$ and the long wave up-welling infrared radiation $\left(R_{l_{-} o}\right)[6]$.

The ground heat flux is based on molecular heat transfer and is proportional to the temperature gradient in the soil $\frac{\Delta T_{s}}{\Delta z}$ and the thermal molecular conductivity $a_{G}$.

The $a_{G}$ values in function of the different types of ground surface can be found in[18]. On summer days, the ground heat is about $50-100 \mathrm{Wm}^{-2}[6]$.

$L E, H, G$ and $R_{n}$ have a totally different representative source areas. $L E$ and $H$ are turbulent fluxes and their representative source area is defined by the footprint size[10]. It depends to the turbulent characteristics of the atmosphere and many other variables for example roughness, wind velocity, measurement height and so on[6]. Its value goes from some meters square to hectares. For $R_{n}$ the representative source area is characterized by the radiometer field of view and it depends, in particular way, on the measurement height. The net radiometer is located on the tower at the height of about 4 meters (see Section 3) and its field of view can be considered of about $50 \mathrm{~m}^{2}$ (for an angle of $45^{\circ}$ ). Ground heat flux $(G)$ is usually very small respect to the other energy fluxes, ranging from 5 to $40 \%$ of net radiation but this flux is the one with the highest uncertainty in its estimate that can reach en error up to $50 \%[6]$. Moreover it is measured with an instrument with the smallest source area that can be up to two orders of magnitude lower than latent and sensible heat fluxes footprints; however, in literature it is assumed that the net radiation and ground heat flux measurements are representative for the total cultivated field[6].

\subsection{The Hsieh's Model}

The Hsieh's model [10] is based on the equation (9).

$$
x=\frac{-1}{k^{2} \ln \left(\frac{F\left(x ; z_{m}\right)}{S_{0}}\right)} D\left(\frac{z_{u}}{|L|}\right)^{P}|L|
$$

$x$ represents the distance from the tower, $k$ is the Von Karman constant and its value is $0.4, D$ and $P$ are called similarity constants and depend on stability conditions of the atmosphere[10]. $z_{u}$ is a length scale and $z_{m}$ represents the height where sensors are installed. $F / S_{0}$ is the ratio between the flux measured by the sensors and the flux emitted by the total field. $S_{0}$ quantity is not known but it is not important because the ratio is always confined from 0 to $1[10]$. $L$ is the Monin - Obukov length[3] and can be calculated by equation (10). 


$$
L=\frac{-u_{*}^{3} \bar{T}}{k g w^{\prime} T^{\prime}}
$$

Where $u *$ is the friction velocity (11), $\bar{T}$ is the mean air temperature, $g$ is the gravity constant and $k$ the Von Karman constant.

$$
u_{*}=\left({\overline{u^{\prime} w^{\prime}}}^{2}+{\overline{v^{\prime} w^{\prime}}}^{2}\right)^{\frac{1}{4}}
$$

Where $\overline{u^{\prime} w^{\prime}}$ and $\overline{v^{\prime} w^{\prime}}$ are the covariance between vertical velocity component and planar (longitudinal and transversal) components of the wind, and $z_{u}$ is defined by (12) where $z_{0}$ represents the surface roughness.

$$
z_{u}=z_{m}\left[\ln \left(\frac{z_{m}}{z_{0}}\right)-1+\frac{z_{0}}{z_{m}}\right]
$$

In growing period it is necessary to change the measurement height $z_{m}$ in $\left(z_{m}-d\right)$ where $d$ is called displacement and it is about $2 / 3$ of $h_{v}[6]$ where $h_{v}$ is the vegetation height. The roughness is calculated by the approximate relation[6] (13).

$$
z_{0}=0.1 h_{v}
$$

\section{Study Sites and Instruments}

Experimental data were obtained by two micrometeorological stations utilized to measure evapotranspiration fluxes in maize fields, one in Landriano (PV) $(45.19 \mathrm{~N}, 9.16$ E, $87 \mathrm{~m}$ a.s.l) and another one in Livraga (LO) $(45.11 \mathrm{~N}, 9.34$ E, $60 \mathrm{~m}$ a.s.l) (Figure 1). The distance between the stations is about $100 \mathrm{Km}$ and they are located at the Po valley.

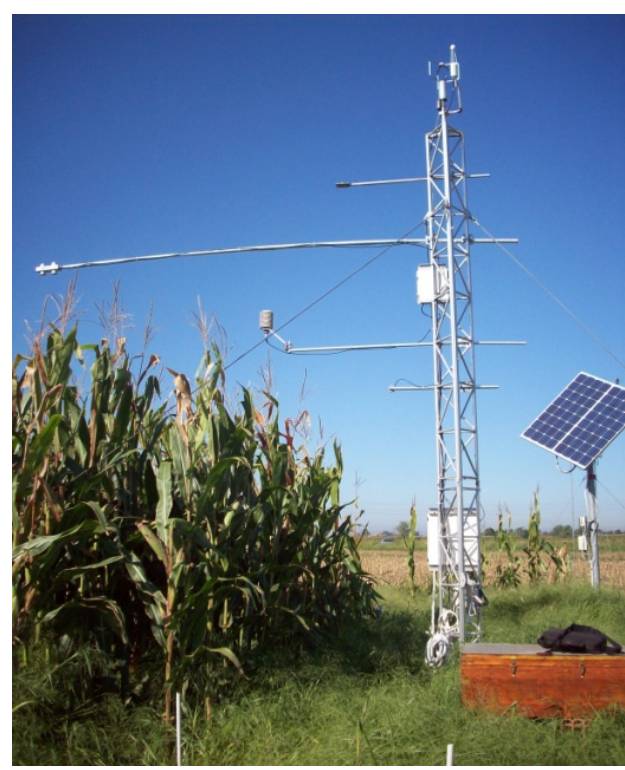

Figure 1. Eddy covariance tower at Livraga (LO).

Both data stations are acquired by a Campbell Scientific data logger (CR 5000) and stored each 30 minutes. The stations are equipped with the following sensors: one 3D sonic anemometer (Young 81000 by Young), that measures air temperature and the three components of wind speed and is positioned at $5 \mathrm{~m}$ height; a gas analyzer (LICOR 7500 by LICOR) for the measurement of air humidity, working with the anemometer at a frequency of $10 \mathrm{~Hz}$, is also positioned at 5m height; a radiometer (CNR1 by Kipp \& Zonen), which measures the four components of net radiation, is positioned at $4 \mathrm{~m}$ height; two thermocouples (by ELSI) and a heat flux plate (HFP01 by Hukseflux) for the measurement of soil ground heat flux positioned respectively at $6-10 \mathrm{~cm}$ and $8 \mathrm{~cm}$; humidity probes (CS616 by Campbell Scientific) for measurement of the volumetric soil moisture at different depths; a pluviometer (AGR100 by Campbell Scientific) at $1.5 \mathrm{~m}$ height.

Data which are used in this work were collected during the year 2010 , from $1^{\text {th }}$ June $\left(152^{\text {th }}\right.$ Julian day) to $10^{\text {th }}$ October $\left(283^{\text {th }}\right.$ Julian day) for Landriano site and from $25^{\text {th }}$ May ( $145^{\text {th }}$ Julian day) to $10^{\text {th }}$ September $\left(253^{\text {th }}\right.$ Julian day) for Livraga site.

Energy fluxes have been corrected applying Webb correction for density fluctuations[22] and the correction for buoyancy flux due to sonic temperature measurements [13].Tilt correction has been applied to take into account that the assumption of a negligible mean vertical velocity is not always verified[19]. Frequency response correction has been applied for the attenuation of eddy covariance fluxes due to sensor response, path-length averaging, sensor separation, signal processing, and flux averaging period[16]. The data during rainfall period were discarded.

The database, after these corrections, is composed of 4163 and for 4507 half hourly data respectively for Landriano and Livraga.

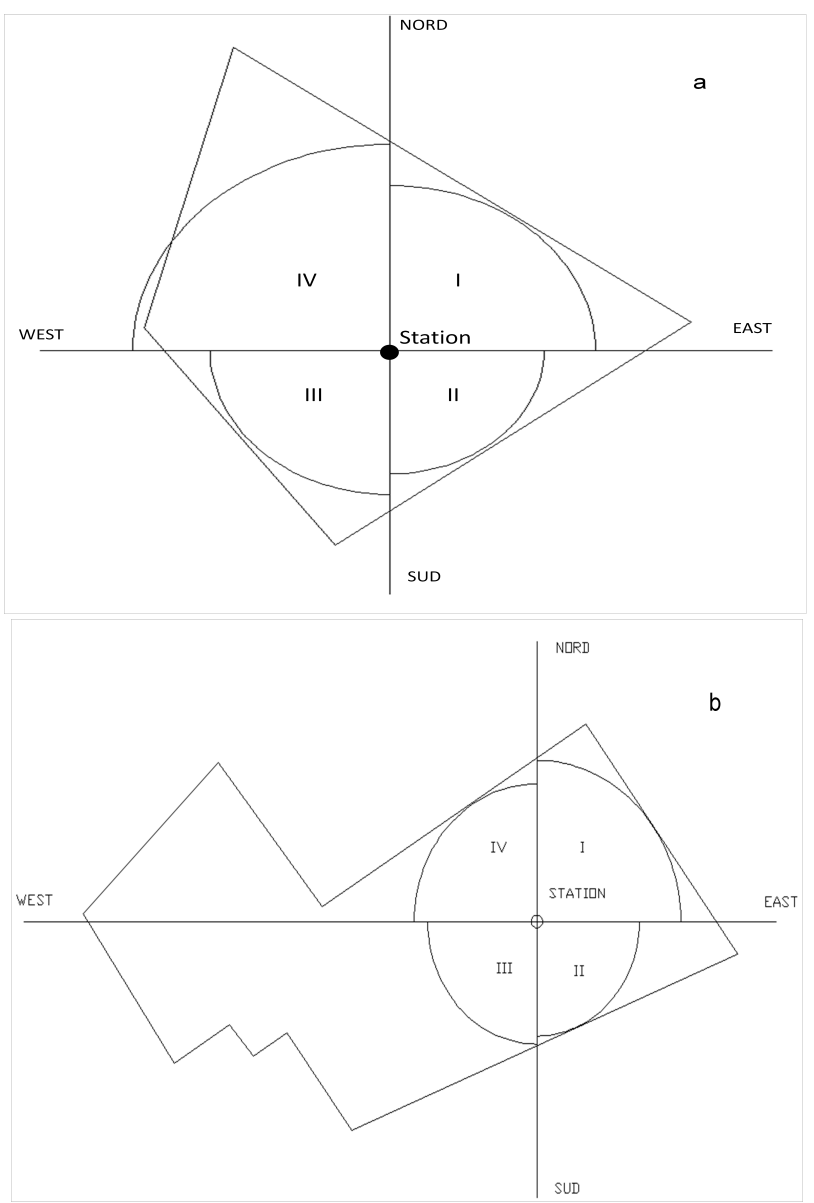

Figure 2. a. Landriano field, b. Livraga field. Subdivision in sectors. 


\section{Method}

The objective of this work is to understand the influence of the representative source area for eddy covariance measurements on energy balance closure.

The first step was to subdivide Landriano and Livraga fields in four different sectors along geographic directions (Figure 2a and $\mathrm{b}$ ) so that the data were subdivided in four classes of 90 degree each depending on wind direction.

For each sector it was definite a radius of a source area which is included at least $95 \%$ into the field. In Table 1 the radius value taken for each sector is shown.

Table 1. Sectors dimension

\begin{tabular}{|c|c|c|}
\hline & \multicolumn{2}{|c|}{ Radius (m) } \\
\hline Sector & Landriano site & Livraga site \\
\hline I & 160 & 195 \\
\hline II & 100 & 90 \\
\hline III & 120 & 120 \\
\hline IV & 200 & 165 \\
\hline
\end{tabular}

For each measurement of latent and sensible heat fluxes was associated the corresponding footprint size using the relation (9) (assuming that $F / S_{0}$ value is equal to 0.8 ), the prevalent wind direction and its sector. It was assumed a value of $F / S_{0}$ equal to $80 \%$ because it represents, in according to the results shown in[10], a typical value over which the footprint shape does not change significantly. The data of latent or sensible heat which had a corresponding source area larger than their respective radius, were deleted.

The energy balance was calculated using equation (6) where $L E, H, R_{n}$ and $G$ were determined by (3), (4), (7) and (8). One main objective of this work is to show how the energy balance closure was influenced by stability conditions of the atmosphere. To define the stability conditions it was taken the stability parameter $\left(z_{u} / L\right)$. If $z_{u} / L<-0.04$ the atmosphere is in unstable conditions; for $z_{u} / L>0.04$ there are stable conditions and for $-0.04<z_{u} / L<0.04$ the atmosphere is in neutral conditions. This classification is, also, important to define the value of $D$ and $P[10]$ in (9). For these different atmospheric situations the slope values of linear regression (m) and linear correlation coefficients $\left(\mathbf{R}^{2}\right)$ for the energy balance closure were calculated. Moreover, it was studied the energy balance closure for different sectors depending on wind direction. For Landriano and Livraga site $L E, H, R_{n}$ and $G$ were collected in four groups: from $0^{\circ}$ to $90^{\circ}$, from $90^{\circ}$ to $180^{\circ}$, from $180^{\circ}$ to $270^{\circ}$, from $270^{\circ}$ to $360^{\circ}$ and for each group the slope of the energy balance closure was calculated. At the same time, it was made a study about source area size and its variability for different atmospheric conditions. A frequency analysis on footprint size was made during instable and neutral conditions because the eddy covariance station works only if there is turbulence into the atmospheric boundary layer[6] and it is guaranteed only in convective (unstable) or neutral conditions[6], instead, in stable conditions the turbulence is formed by eddies which have small dimension (some centimetres) and are originated by the mechanical forces of the wind shear[24]. In these cases the atmosphere is quite similar to a laminar structure. For each sector the footprint values were subdivided in twenty classes and subsequently the number of footprint values which fallen in each class was calculated. It was also shown the percentage of data which had to be deleted because the footprint size was larger than field dimension.

\section{Results and Discussion}

The results show that the slope of the energy balance closure increases slightly if the data without a footprint size compatible with the field dimension were deleted. In Figure 3 and 4 are shown the energy balance closure respectively for Landriano and Livraga site. In Figure $3 \mathrm{a}$ and $4 \mathrm{a}$ are represented the energy balance closure for all data set, while in Figure $3 b$ and $4 b$ the energy balance closure is calculated using only the footprint compatible data. For Landriano site, the slope of the linear regression has a slight improvement of about $0.40 \%$ and for the linear correlation coefficient of about $3.8 \%$. For Livraga site, the difference in slope is $0.33 \%$ and in $\mathrm{R}^{2}$ is equal to $4.24 \%$

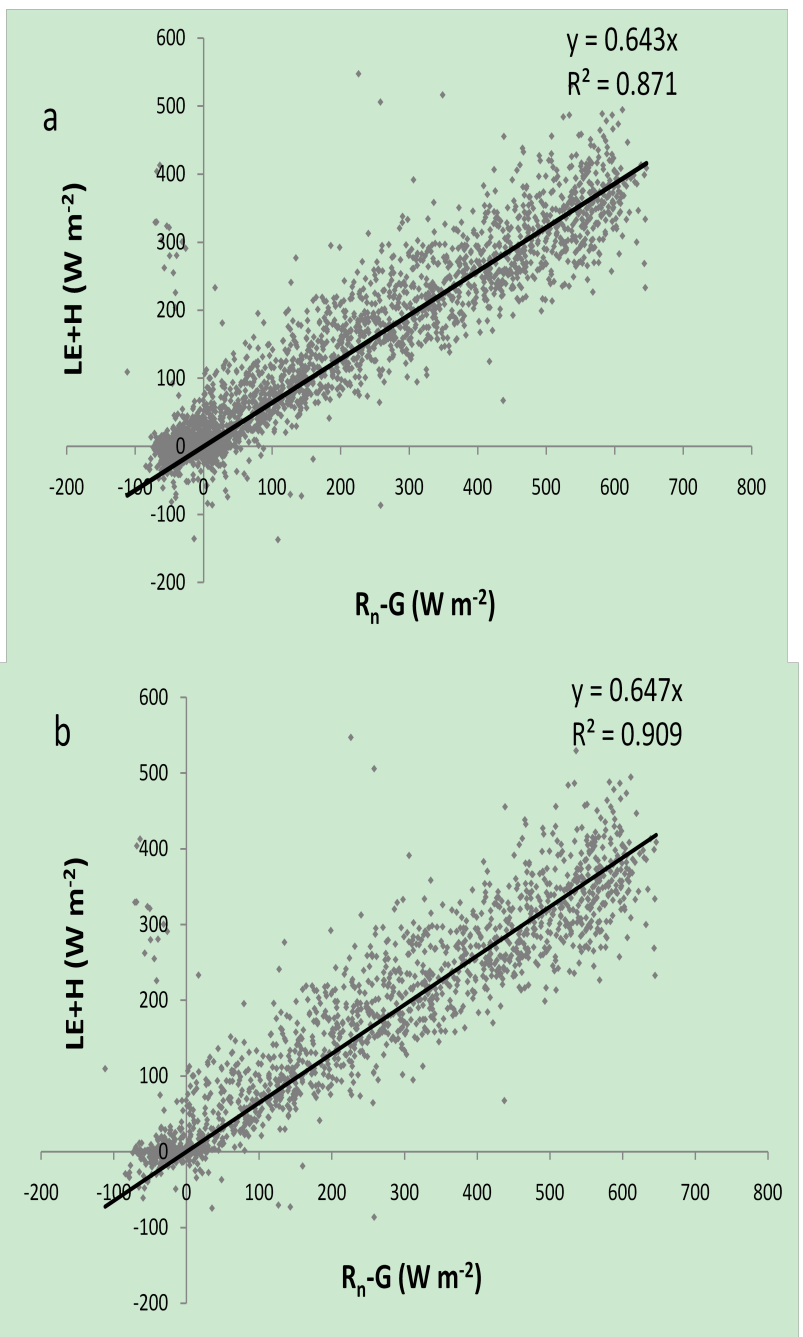

Figure 3. Landriano site. a. energy balance closure with all data. b. energy balance closure using the data with a footprint compatible with field dimension. 
Considering only the data which have a footprint size compatible with the fields dimension, for Landriano site, the number of data changes from 4163 to 2329 , which represents about $55 \%$ over total data set. For Livraga site, the number of data changes from 4507 to 2401 which represents about 53\% over total data set.

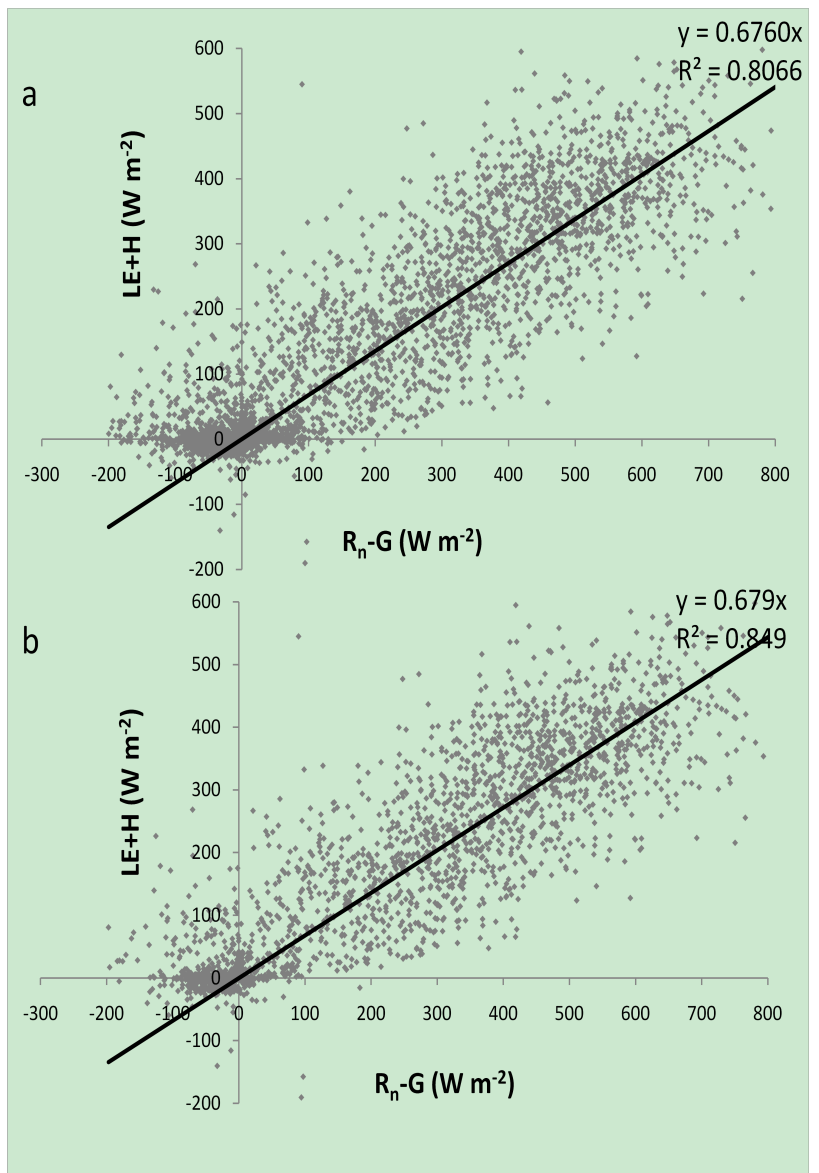

Figure 4. Livraga site. a. energy balance closure with all data. b. energy balance closure using the data with a footprint compatible with field dimension.
In the Po Valley the geometric structure of the fields have a polygonal shape and there are irrigation ditches around them. Landriano and Livraga fields are surrounded by other maize fields and it is probable that the slight improvement of the linear regression slopes is caused by the quite similar evapotranspiration among the fields. Moreover, the fields dimensions are smaller than those in USA country[1]. In fact Landriano and Livraga fields are about 10 hectare large. For example in Boardman, OR (USA), the fields have a circular shape and they are surrounded by the desert. In these cases the spatial variability of surface fluxes is very big, in fact in [1] is shown that the variation of measured latent heat flux densities across a potato field from discontinuity point (desert-field) to the centre of the field is about $60 \%$.

The stability conditions of the atmosphere play an important role on the energy balance closure improvement. In Table 2 and 3 the values of $\mathbf{m}$ and $\mathbf{R}^{\mathbf{2}}$ are shown for different stability conditions of the atmosphere (stable, neutral and unstable conditions). The value of $\mathbf{m}$ shown in Table 2 and III confirmed that in unstable and neutral conditions the heat fluxes measured by the eddy tower are correct while in stable conditions the heat fluxes are incorrect because the turbulence structure into the atmospheric boundary layer is not well definite[24].

So, in additions to the flux corrections indicated in Section 2 the flux data measured in stable conditions have to be deleted; in these cases it is nonsense to talk about representative source area for turbulent heat fluxes. For this reason in Table 2 and 3 the value of the slope and $\mathbf{R}^{2}$ in "fluxes with footprint" are not indicated.

The footprint dimension is a main feature which has to be used to choose the correct turbulent flux data. In unstable and neutral conditions the representative source area for latent and sensible heat fluxes can be larger than field dimension. In these cases the flux data have to be deleted. The effect of the data removal is shown in Table 2 and 3 with reference to "Fluxes with footprint" and " $N^{\circ}$ data with footprint".

Table 2. Landriano site. Slope values of the linear regression $(\mathbf{m})$ and linear correlation coefficient $\left(\mathbf{R}^{2}\right)$ for different stability conditions of the atmosphere for all data set and for only the data which have a footprint size compatible with field dimension.

\begin{tabular}{|c|c|c|c|c|c|c|c|c|}
\cline { 2 - 10 } \multicolumn{1}{c|}{} & \multicolumn{2}{c|}{ Total fluxes } & \multicolumn{2}{c|}{ Fluxes with footprint } & $\begin{array}{c}\mathbf{N}^{\circ} \\
\text { data }\end{array}$ & $\begin{array}{c}\text { \%o of } \\
\text { data }\end{array}$ & $\mathbf{N}^{\circ}$ data with footprint & \% data with footprint \\
\cline { 2 - 10 } \multicolumn{1}{c|}{} & $\mathbf{m}$ & $\mathbf{R}^{\mathbf{2}}$ & $\mathbf{m}$ & $\mathbf{R}^{\mathbf{2}}$ & & & & \\
\hline Unstable conditions & 0.6453 & 0.9045 & 0.6467 & 0.9097 & 1980 & 47.6 & 1840 & 44.2 \\
\hline Neutral conditions & 0.6552 & 0.9035 & 0.6609 & 0.9478 & 977 & 23.5 & 477 & 11.5 \\
\hline Stable conditions & 0.1415 & 0.0744 & & & 1206 & 29.0 & & \\
\hline Total data & 0.6433 & 0.8715 & 0.6473 & 0.9095 & 4163 & 100.0 & 2329 & 55.9 \\
\hline
\end{tabular}

Table 3. Livraga site. Slope values of the linear regression $(\mathbf{m})$ and linear correlation coefficient $\left(\mathbf{R}^{2}\right)$ for different stability conditions of the atmosphere for all data set and for only the data which have a footprint size compatible with field dimension.

\begin{tabular}{|c|c|c|c|c|c|c|c|c|}
\cline { 2 - 9 } \multicolumn{1}{c|}{} & \multicolumn{2}{c|}{ Total fluxes } & \multicolumn{2}{c|}{ Fluxes with footprint } & $\begin{array}{c}\mathbf{N}^{\circ} \\
\text { data }\end{array}$ & $\begin{array}{c}\text { \% of } \\
\text { data }\end{array}$ & $\mathbf{N}^{\circ}$ data with footprint & $\begin{array}{c}\text { \% data with foot- } \\
\text { print }\end{array}$ \\
\cline { 2 - 10 } & $\mathbf{m}$ & $\mathbf{R}^{\mathbf{2}}$ & $\mathbf{m}$ & $\mathbf{R}^{\mathbf{2}}$ & & & & \\
\hline Unstable conditions & 0.6790 & 0.8750 & 0.6770 & 0.8820 & 1725 & 38.3 & 1611 & 35.7 \\
\hline Neutral conditions & 0.7050 & 0.8220 & 0.6980 & 0.8420 & 1276 & 28.3 & 893 & 19.8 \\
\hline Stable conditions & 0.0290 & 0.0070 & & & 1386 & 30.8 & & \\
\hline Total data & 0.6760 & 0.8070 & 0.6790 & 0.8490 & 4507 & 100.0 & 2401 & 53.3 \\
\hline
\end{tabular}


The slight improvement on $\mathbf{m}$ and $\mathbf{R}^{\mathbf{2}}$ in function of the different stability conditions of the atmosphere (unstable and neutral) is shown in Table 4. While in Landriano site in unstable and neutral conditions the slope of the linear regression for the energy balance closure (from "Total fluxes" to "Fluxes with footprint") increase its value, in Livraga site it decreases. However, using the total data set which is also composed by data measured in stable conditions, the slight improvement on $\mathbf{m}$ value for Livraga site is guaranteed how shown in Table 2 and 3. The dispersion of the points around the linear regression curve is minimized; in fact the value of $\mathbf{R}^{2}$ increases in Landriano and Livraga site for unstable and neutral conditions.

Table 4. Energy balance closure improvement on $\mathbf{m}$ and $\mathbf{R}^{2}$ for different conditions of the atmosphere, for Landriano and Livraga site.

\begin{tabular}{|c|c|c|c|}
\cline { 3 - 4 } \multicolumn{2}{c|}{} & $\begin{array}{c}\text { Unstable } \\
\text { conditions }\end{array}$ & $\begin{array}{c}\text { Neutral } \\
\text { conditions }\end{array}$ \\
\hline Landriano & Improvement on $\mathbf{m}(\%)$ & 0.14 & 0.57 \\
\hline & Improvement on $\mathbf{R}^{\mathbf{2}}(\%)$ & 0.52 & 4.43 \\
\hline Livraga & Improvement on $\mathbf{m}(\%)$ & -0.20 & -0.70 \\
\hline & Improvement on $\mathbf{R}^{\mathbf{2}}(\%)$ & 0.70 & 2.00 \\
\cline { 2 - 4 }
\end{tabular}

\subsection{Energy Balance Closure for Field Sectors}

In Figure 5 and 6 the energy balance closure and the

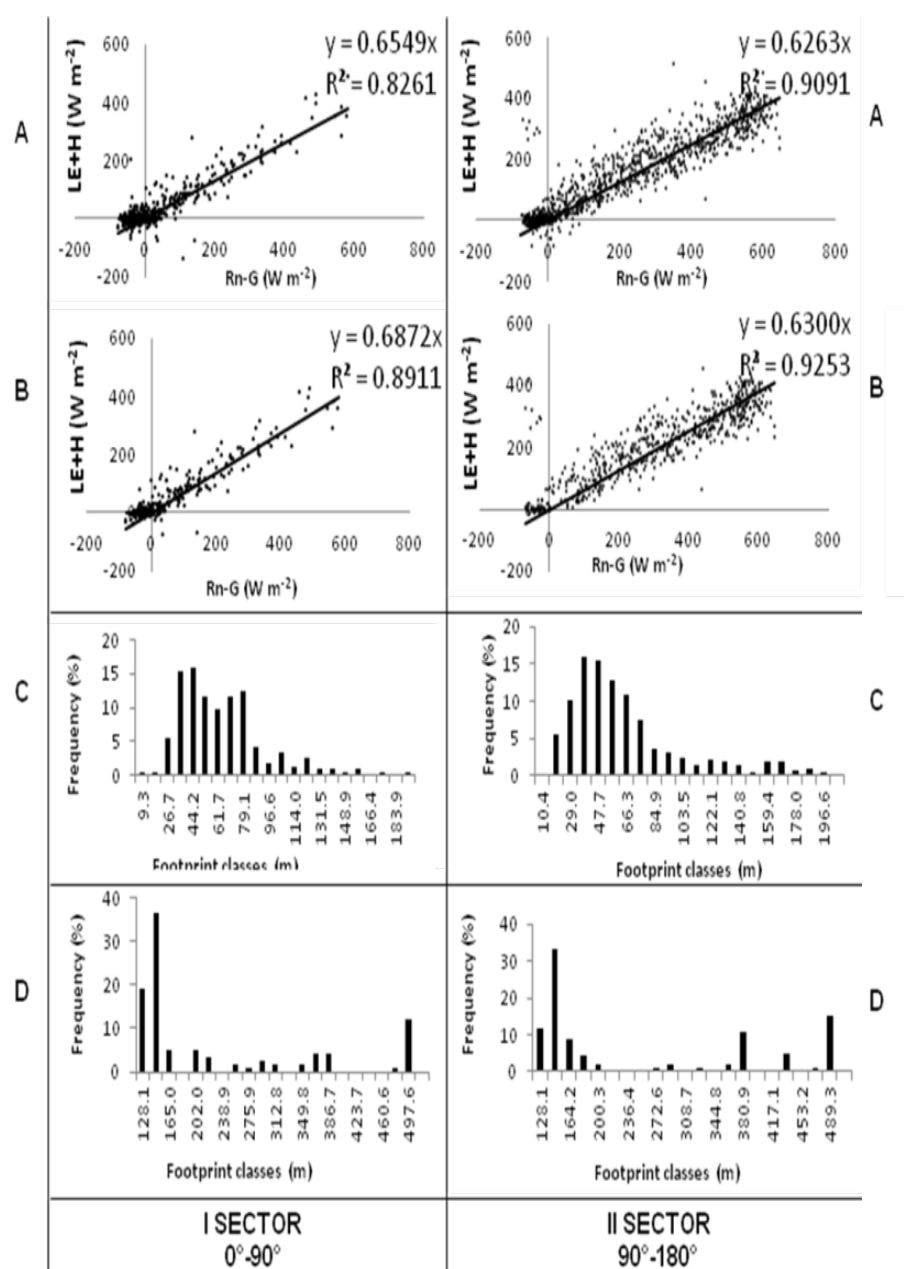

footprint size for the different field sectors as a function of the wind directions are shown. In Figure 5A and 6A the energy balance closure taking into account all data set is represented, while in Figure 5B and 6B the energy balance closure is made using only the data with a footprint length compatible with the field dimension. The energy balance closure characterization for different sectors is necessary to understand how each sector field takes part on total energy balance closure. In fact in some cases it is present an $\mathbf{m}$ value improvement (sectors I, II and IV for Landriano, and sectors I, III and IV for Livraga) but in other cases the $\mathbf{m}$ value decrease (sector III for Landriano and sector II for Livraga).

In Table 5 the percentages of improvement of the slope of linear regression and $\mathbf{R}^{\mathbf{2}}$ for the data which have a footprint size included into the field are represented. In general in Landriano and Livraga sites for almost all the sectors a slight improvement in both $\mathbf{m}$ and $\mathbf{R}^{2}$ values is presented. In Figure 5 and $6(\mathrm{C}$ and $\mathrm{D})$ the footprint sizes for different classes subdivided in unstable and neutral conditions of the atmosphere are represented. During convective conditions the source area lengths are distributed over all the footprint classes, while in neutral conditions only some classes have the most part of the footprint values.

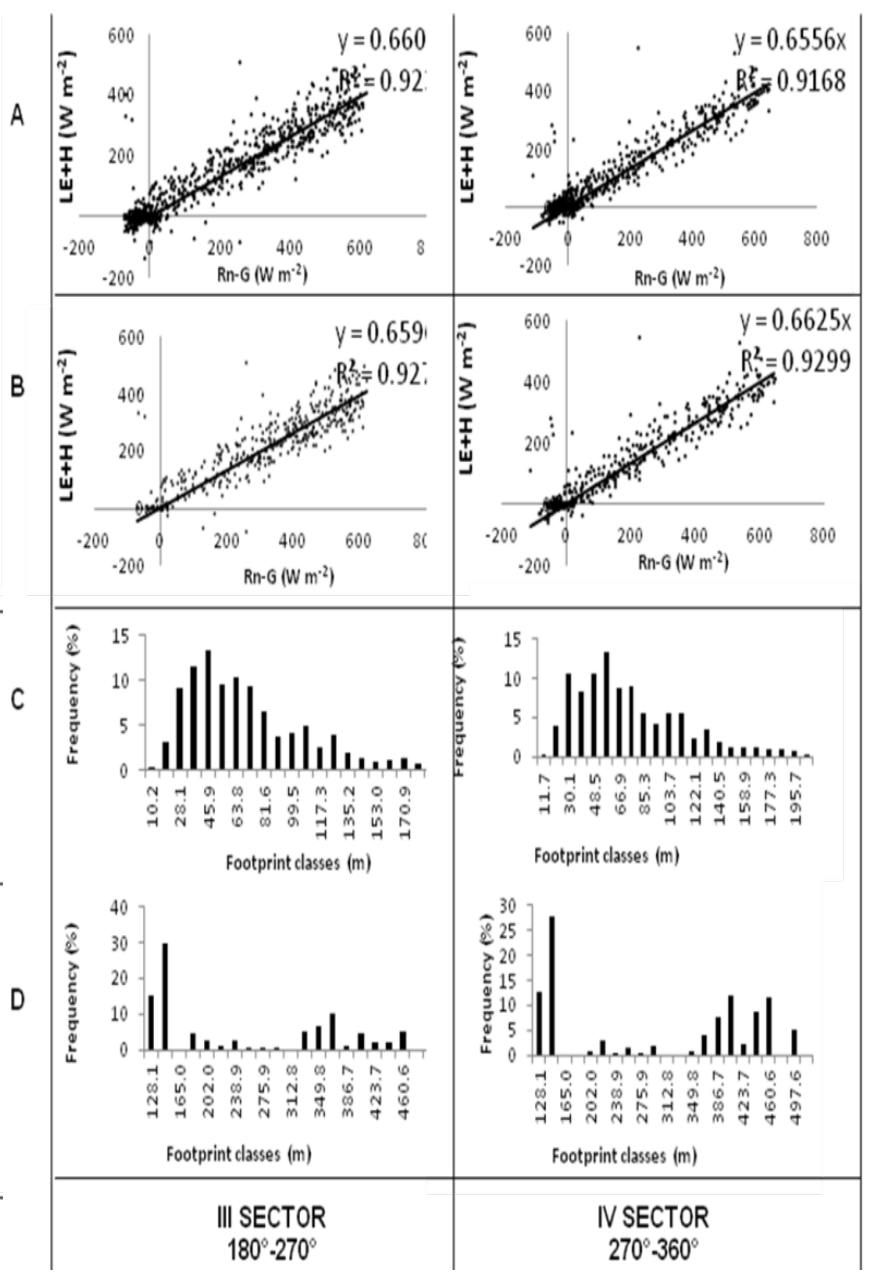

Figure 5. Landriano site. A. Energy balance closure with all data. B. Energy balance closure using the data which have a footprint size less than field dimension. C. Footprint length for unstable conditions of the atmosphere. D. Footprint length for neutral conditions of the atmosphere. 

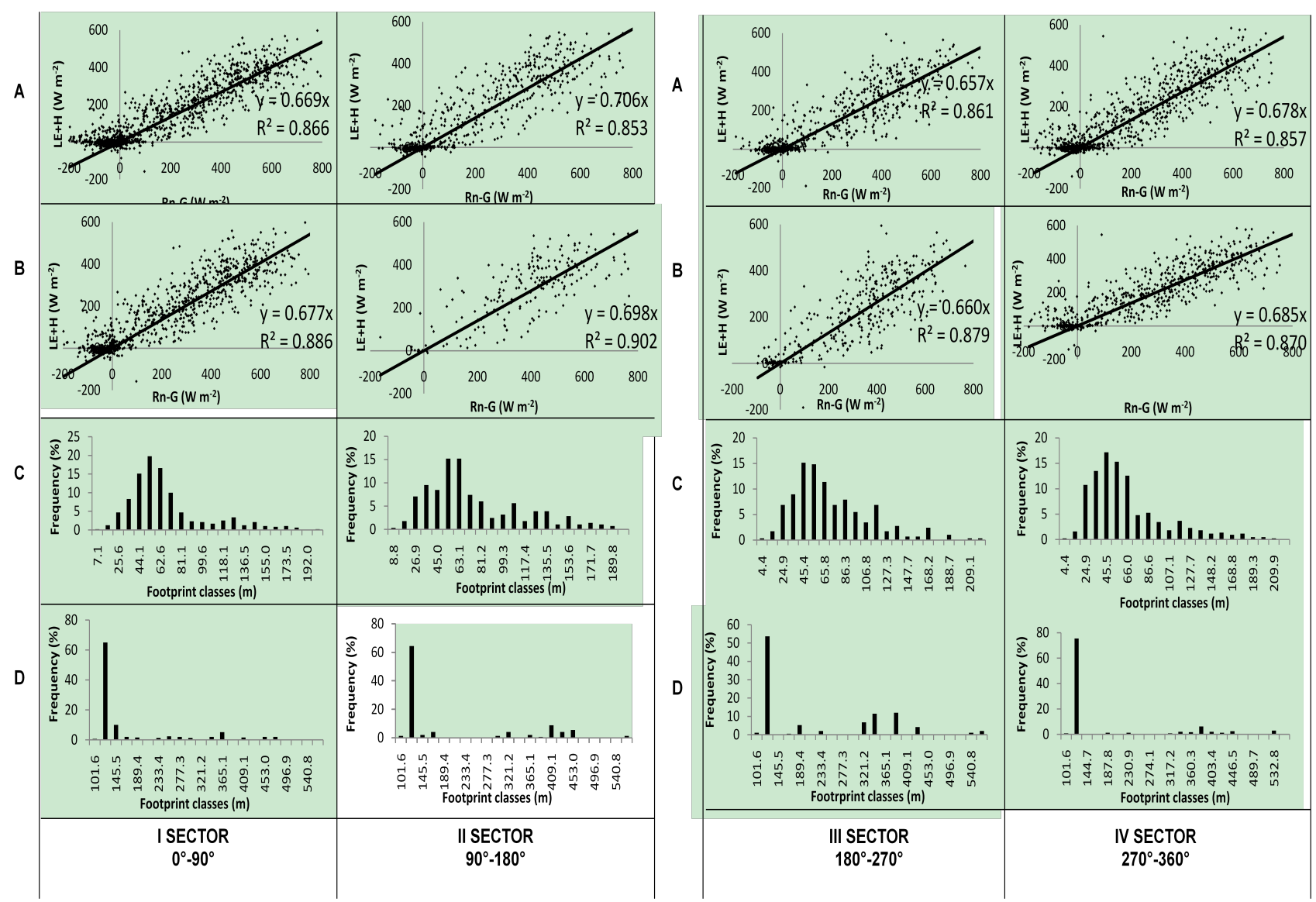

Figure 6. Livraga site. A. Energy balance closure with all data. B. Energy balance closure using the data which have a footprint size less than field dimension. C. Footprint length for unstable conditions of the atmosphere. D. Footprint length for neutral conditions of the atmosphere.

Table 5. Energy balance closure improvement on $\mathbf{m}$ and $\mathbf{R}^{2}$ for each sector, for Landriano and Livraga site.

\begin{tabular}{|c|c|c|c|c|c|}
\cline { 3 - 5 } \multicolumn{1}{c|}{} & I SEC. & II SEC. & IIISEC. & IVSEC. \\
\hline Landriano & Improvement on $\mathbf{m}(\%)$ & 3.23 & 0.37 & -0.040 & 0.69 \\
\hline & Improvement on $\mathbf{R}^{\mathbf{2}}(\%)$ & 6.50 & 1.62 & 0.44 & 1.31 \\
\hline Livraga & Improvement on $\mathbf{m}(\%)$ & 0.83 & -0.75 & 0.36 & 0.68 \\
\hline & Improvment on $\mathbf{R}^{\mathbf{2}}(\%)$ & 1.99 & 4.87 & 1.79 & 1.3 \\
\hline
\end{tabular}

Table 6. Footprint data which have a length less than the field dimension.

\begin{tabular}{|c|c|c|c|c|c|}
\cline { 3 - 5 } \multicolumn{2}{c|}{} & I SEC. & II SEC. & IIISEC. & IVSEC. \\
\hline Landriano & Unstable conditions (\%) & 99.10 & 84.80 & 88.60 & 100.00 \\
\hline & Neutral conditions (\%) & 55.60 & 0.00 & 0.00 & 40.40 \\
\hline Livraga & Unstable conditions (\%) & 99.70 & 73.40 & 90.00 & 96.70 \\
\hline & Neutral conditions (\%) & 79.20 & 0.00 & 54.60 & 76.70 \\
\hline
\end{tabular}

In Table 6 the percentage of the footprint data with a length less than the radius is shown. Only during the convective conditions, the footprint size is almost compatible with the field dimension and the percentage of data which can be used is $>80 \%$. Instead, in some cases where there are neutral conditions of the atmosphere the footprint size is completely larger than field dimension and the flux data can not be used.

\section{Conclusions}

In this work the effect of the source area of eddy covariance measurements on energy balance closure for maize fields in Po Valley has been shown. The energy balance was evaluated for different conditions of the atmosphere and to understand their effects on the closure, the slope of the linear regression (m) and the linear correlation coefficient $\left(\mathbf{R}^{2}\right)$ were calculated. The results show that the eddy covariance stations works only in unstable and neutral conditions. In stable conditions the atmospheric boundary layer structure is quite laminar and the turbulent heat fluxes measured by the eddy tower are uncorrected. During neutral conditions, a lot of data have to be deleted because the turbulent fluxes source representative area exceeds the field dimension. In Landriano and Livraga site the effect of the representative source area for eddy fluxes leads to a slight improvement into the energy balance closure, instead, the stability conditions of the atmosphere play a fundamental role for the determination 
of the slope of the linear regression curve. The study on the representative source area for eddy covariance measurements is important to understand if the station position into the field is correct, the energy balance closure makes for different sector in function of the wind directions shows that in Landriano and Livraga sites the eddy covariance towers are located correctly and the latent and sensible heat measured are representative of the maize fields.

\section{ACKNOWLEDGES}

This work was funded in the framework of the ACQWA EU/FP7 project (grant number 212250) "Assessing Climate impacts on the Quantity and quality of WAter", the framework of the ACCA project funded by Regione Lombardia "Misura e modellazione matematica dei flussi di ACqua e CArbonio negli agro-ecosistemi a mais" and PREGI (Previsione meteo idrologica per la gestione irrigua) founded by Regione Lombardia. The authors thank the University of Milan - Dipartimento di Idraulica Agraria for the collaboration given in managing Landriano eddy covariance station.

\section{REFERENCES}

[1] Baldocchi, D. and Rao, K., 1995. Intra-field variability of scalar flux densities across a transition between desert and an irrigated potato field. Boundary Layer Meteorology, 76, $109-136$

[2] Barr, A., Morgenstern, K., Black, T., McCaughey, J. and Nesic, Z., 2006. Surface energy balance closure by the eddy-covariance method above three boreal forest stands and implications for the measurement of $\mathrm{CO} 2$ flux. Agricultural and Forest Meteorology , 140, 322-337

[3] Calder, K., 1965. Concerning the similarity theory of A. S. Monin and A. M. Obukhov for the turbulent structure of the thermally stratified surface layer of the atmosphere. Quarterly Journal of the Royal Meteorological Society , 92, 141-146

[4] Culf, A., Foken, T. and Gash, J., 2004. The energy balance closure problem, in: A new prospective on an iteractive system. Kabat, P., Claussen ,M., Vegetation, Water, Humans and Climate, Springer, Berlin, 159-166

[5] Dyer, A., 1963. The adjustament of profiles and eddy fluxes. Quart. J. R. Meteorol.Soc. ,89, 276-280

[6] Foken, T., 2008. Micrometeorology. Springer, Berlin, 306

[7] Foken, T., Wimmer, F., Mauder, M., Thomas, C. and Liebhetal, C., 2006. Some aspects of the energy balance closure problem. Atmos. Chem. Phys. , 6, 4395-4402

[8] Gash, J., 1985. A noteon estimating the effect of a limited fetch on micrometeorological evaporation measurements. Boundary Layer Meteorology , 35, 409-413

[9] Hipps, L., Prueger, J., Eichinger, W. and Kustas, W. Relations between enviormental conditions and the ability to close energy balance. In Proceedings of the 27th Conference on
Agricoltural and Forest Meteorology, May 22-25, 2006, San Diego, CA. 2006 CDROM

[10] Hsieh, C., Katul, G. and Chi, T., 2000. An approximate analytical model for footprint estimation of scalar fluxes in thermally stratified atmospheric flows. Advanced in water researc , 23, 765-772

[11] Hsieh, C., Katul, G., Schieldge, J., Sigmon, J. and Knoerr, K., 1997. The Lagrangian stochastic model for fetch and latent heat flux estimation above uniform and nonuniformterrain. Water Resources Research , 33, 427-438

[12] Kljun, N., Rotach, M. and Schmid, H., 2002. A 3D bacward Lagrangian footprint model for a wide range of buondary layer stratifications. Boundary Layer Meteorology , 103, 205-226

[13] Liu, H., Peters, G., \& Foken, T. (2001). New equations for sonic temperature variance and buoyancy heat flux with an omnidirectional sonic anemometer. Bound. Layer. Meteorology, 100, 459-468

[14] Mao, S., Feng, Z. and Michaelides, E., 2007. Large-eddy simulation of low-level jet-like flow in a canopy. Envaiormental Fluid Mechanics , 7, 73-93

[15] Markkanen, T., Steinfeld, G., Kljun, N., Raasch, S., and Foken, T. (2009). Comparison of conventional Lagrangian stochastic footprint models against LES driven footprint estimates. Atmospheric Chemistry and Physics, 9, 5575-5586

[16] Massman, W. and Lee, X., 2002. Eddy covariance flux corrections and uncertainties in long-term studies of carbon and energy exchanges. Agricoltural Forest Meteorology, 113, $121-144$

[17] Schmid, H., 2002. Footprint modeling for vegetation atmosphere exchange studies: a review and prospective. Agricoltural Forest Meteorology , 113, 159-184

[18] Stull, R., 1988. An introduction to boundary layer meteorology. Dordrecht, Boston, London, 666. Kluwer Academic Publisher

[19] Tanner, C. and Thurtell, G., 1969. Anemoclinometer measurements of Reynolds stress and heat transport in the atmospheric surface layer. ECOM 66-G22-F, ECOM, United States Army Eletronics Command, Research and Developement

[20] Thomson, D., 1978. Criteria for the selection of stochastic models of particle trajectories in turbolent flows. Journal of Fluid Mechanic , 180, 529-56

[21] Van Ulden, A., 1978. Simple estimates for vertical diffusion from cources near the ground. Atmospheric Enviorment , 12, $2125-2129$

[22] Webb, E., Pearman, G. and Leuning, R., 1980. Correction of the flux measurements for density effects due to heat and water vapour transfer. Boundary Layer Meteorology, 23, 251-254

[23] Wilson, K., Goldstein, A., Falge, E., Aubinet, M., Baldocchi, D., Berbigier, P., et al. (2002). Energy balance closure at FLUXNET sites. Agricoltural Forest Meteorology, 113, 223-243

[24] Wyngaar, J., 1990. Scalar fluxes in the planetary boundary layer. Boundary Layer Meteorology, 50, 49-79 